\title{
Sonographic Diagnosis of Ovarian Masses
}

\author{
Kohkichi Hata \\ Department of Nursing, Kagawa Prefectural University of Health Sciences, Takamatsu, Japan \\ Correspondence: Kohkichi Hata, Department of Nursing, Kagawa Prefectural University of Health Sciences, \\ 281-1 Murecho-hara, Takamatsu 761-0123, Japan \\ Telephone: +81-87-870-1578, Fax: +81-87-870-1204, e-mail: hata@chs.pref.kagawa.jp
}

\begin{abstract}
Transabdominal ultrasonography was initially used for the investigation of overt abdominal swellings and much later for the detection of early ovarian cancer in asymptomatic women. The technique was undoubtedly of value for detecting transient, persistent and overt ovarian cysts. Subsequently, the resolving power of pelvic ultrasonography was increased substantially by the advent of transvaginal probes. This revolutionary development was first applied to systemic studies of overt ovarian masses in patients awaiting surgery. Over the past decade, several ultrasound-based morphological scoring systems, color Doppler parameters, logistic regression models have been proposed and tested in order to try to predict the histology of ovarian tumors. On prospective testing, no standard criterion has been published. Therefore, it is important for each operator to establish their own criteria based on individual recognition potential.
\end{abstract}

Key words: Ultrasonography, ovarian masses, morphological scoring systems, color Doppler imaging with pulsed Doppler spectral analysis, multivariate logistic regression analysis, pattern recognition.

\section{INTRODUCTION}

Ovarian lesions are of particular importance in gynecological practice owing to their malignant potential and the limited ability to differentiate between benign and malignant types prior to surgery. Therefore, the preoperative diagnosis of ovarian tumor with B-mode ultrasonography has been very important. As the pelvis contains many soft tissue organs with a similar acoustic impedance, previous studies employing transabdominal sonography could not provide a clear image of the various structures due to low resolution, unacceptable attenuation of the transmitted pulses (and the reflected echoes), and particularly in obese patients, due to the ovaries being well outside the focal region of the transducer. Therefore, the anticipation of ultrasound-derived endpoints which could be used to distinguish malignant from benign cysts proved to be premature. ${ }^{1}$ By the advent of transvaginal transducer with high frequency, the better resolution as compared to abdominal transducer, we were able to depict a more detailed imaging of female intrapelvic organs, especially normal ovaries (Figs 1 and 2). This revolutionary development was soon applied to systematic studies of overt ovarian masses in patients awaiting
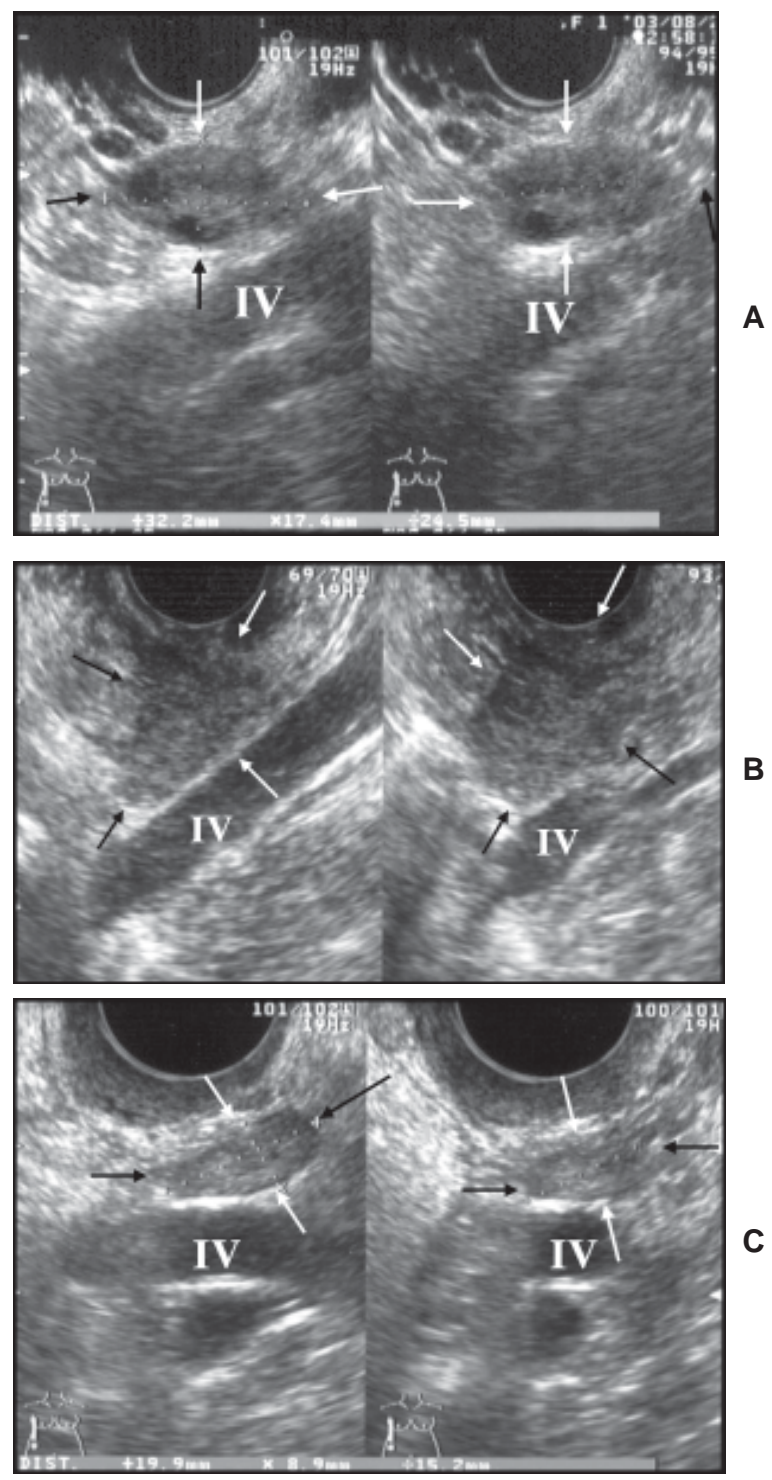

Fig. 1: Transvaginal sonography of normal ovary (arrows) (A) premenopausal, (B) artificial postmenopausal, (C) postmenopousal, IV: iliac vessel) 


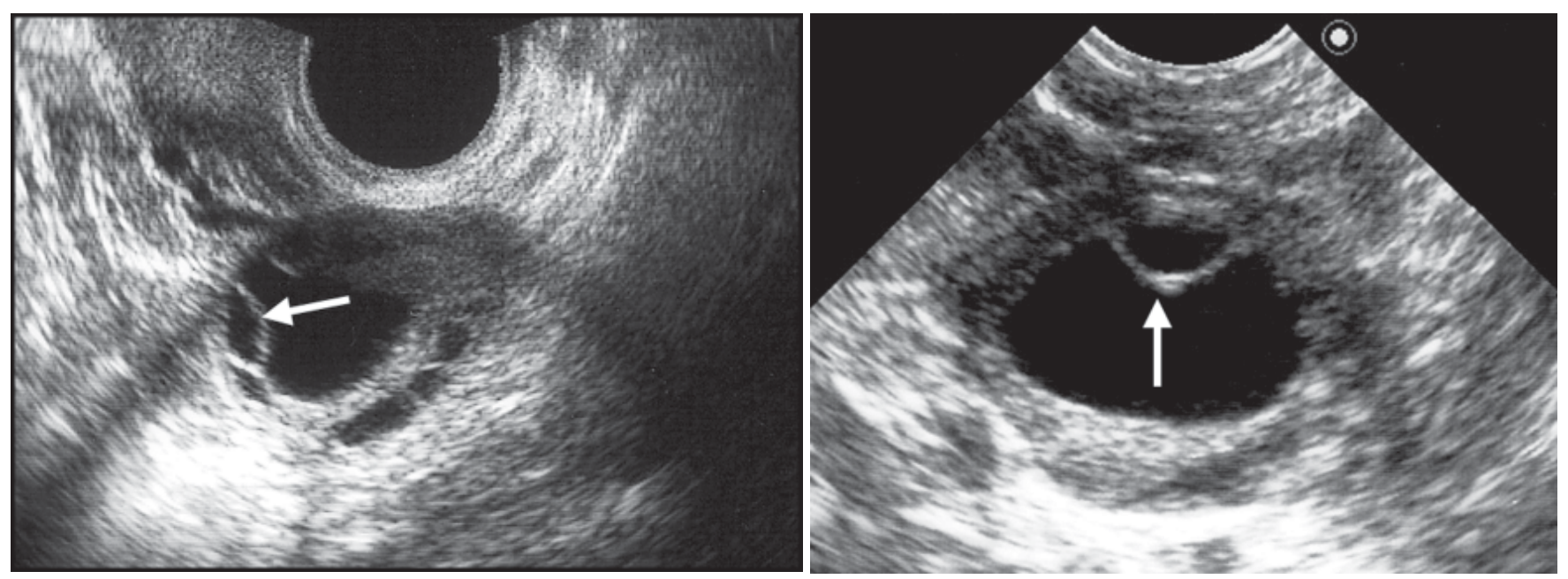

A
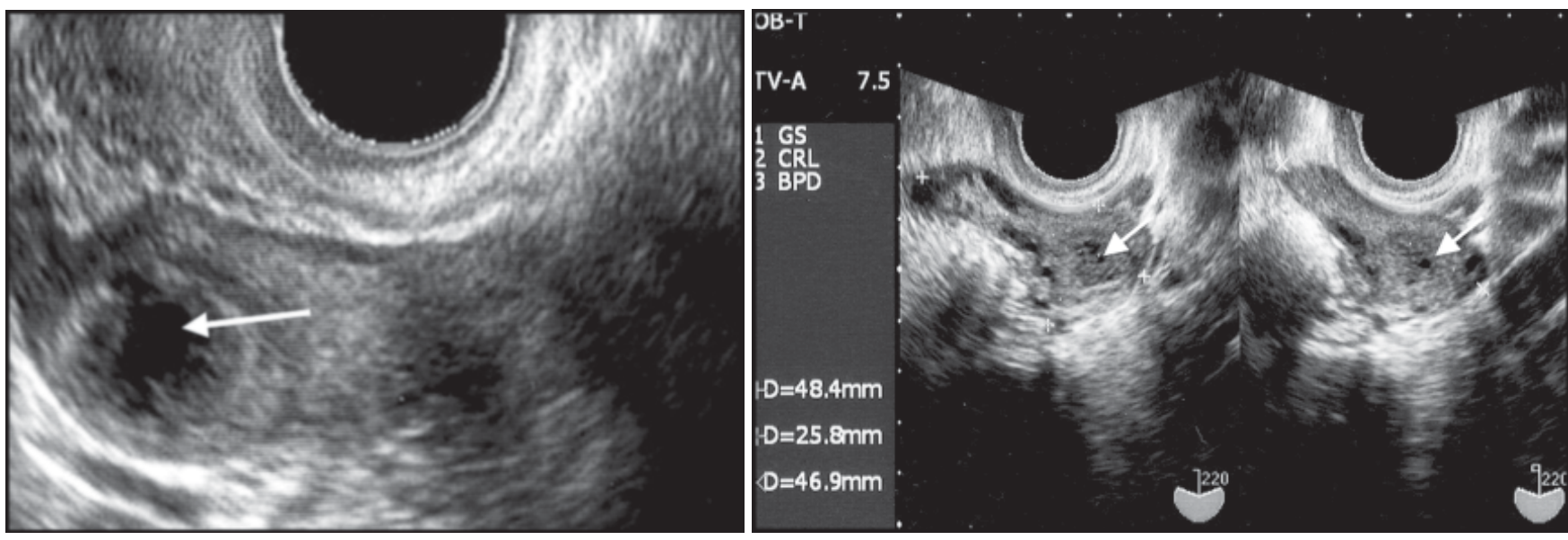

B
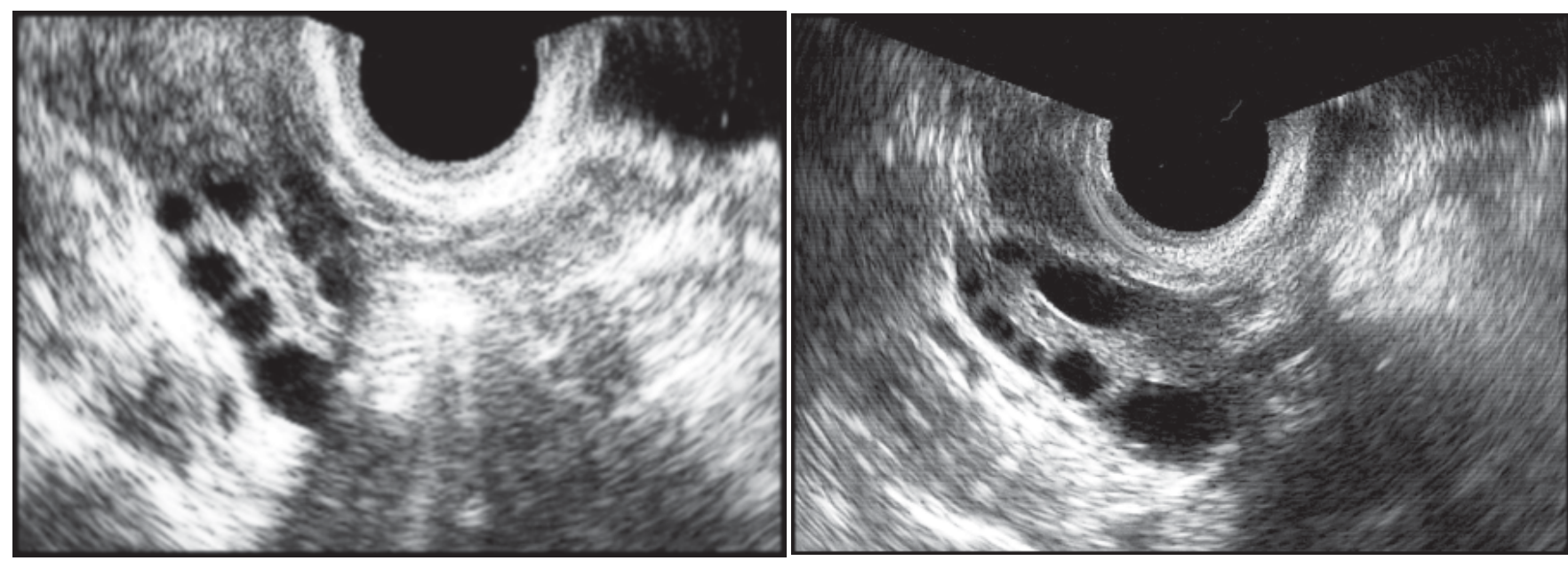

C

Fig. 2: Physiological change of appearance in normal ovary: (A) cumulus oophorus formation (arrow), (B) corpus luteum formation (arrow), (C) polycystic ovary 
surgery. Moreover, the introduction of transvaginal color Doppler imaging with pulsed Doppler spectral analysis represented a quantum leap in technical development. This additional facility enabled studies to be undertaken on the vascularity and blood flow within simple and complex forms of ovarian cyst. ${ }^{2}$

This time, I review the usefulness and limitation of sonographic evaluation of ovarian masses. I hope this review will be useful in clinical practice.

\section{OBJECTIVE EVALUATION \\ Transvaginal B-mode Diagnosis}

Granberg et $a l^{3}$ and Rottem et $a l^{4}$ established sonographic criteria of ovarian tumors, which could be used to classify a tumor as benign (i.e. the cyst must be unilocular and thin-walled with no irregularities in outline or expanding processes or septa, or echogenicity) or malignant (i.e. the ovary must be multicystic, or a single cyst must be multilocular with dense or irregular septa, have a poorly defined border with papillary formation on the inner surface, or increased fluid in the peritoneal cavity). In our opinion, although the criteria are objective and acceptable, an increase in false-negative results will be inevitable. Only adnexal tumor of unilocular cystic pattern is diagnosed to be benign (Fig. 3). This is the limitation of B-mode ultrasonography with ovarian tumor based on macroscopic pathology. However, the consensus view is that transvaginal B-mode ultrasonography is sensitive to detect nearly all cystic ovarian lesions suggestive of carcinoma (and hence increases false-positive rate).

Several morphological scoring systems have been developed and examined by different centers to improve the accuracy of ultrasound diagnosis ${ }^{5-7}$ (Fig. 4). Initial results encouraged a systematic examination of a number of different tumor features on ultrasound scan. However, none of these scoring systems has been widely adopted in routine clinical practice. Because, each author had no information regarding the reproducibility of the scoring system, and the relatively small number of subjects precluded a precise estimate of test performance.
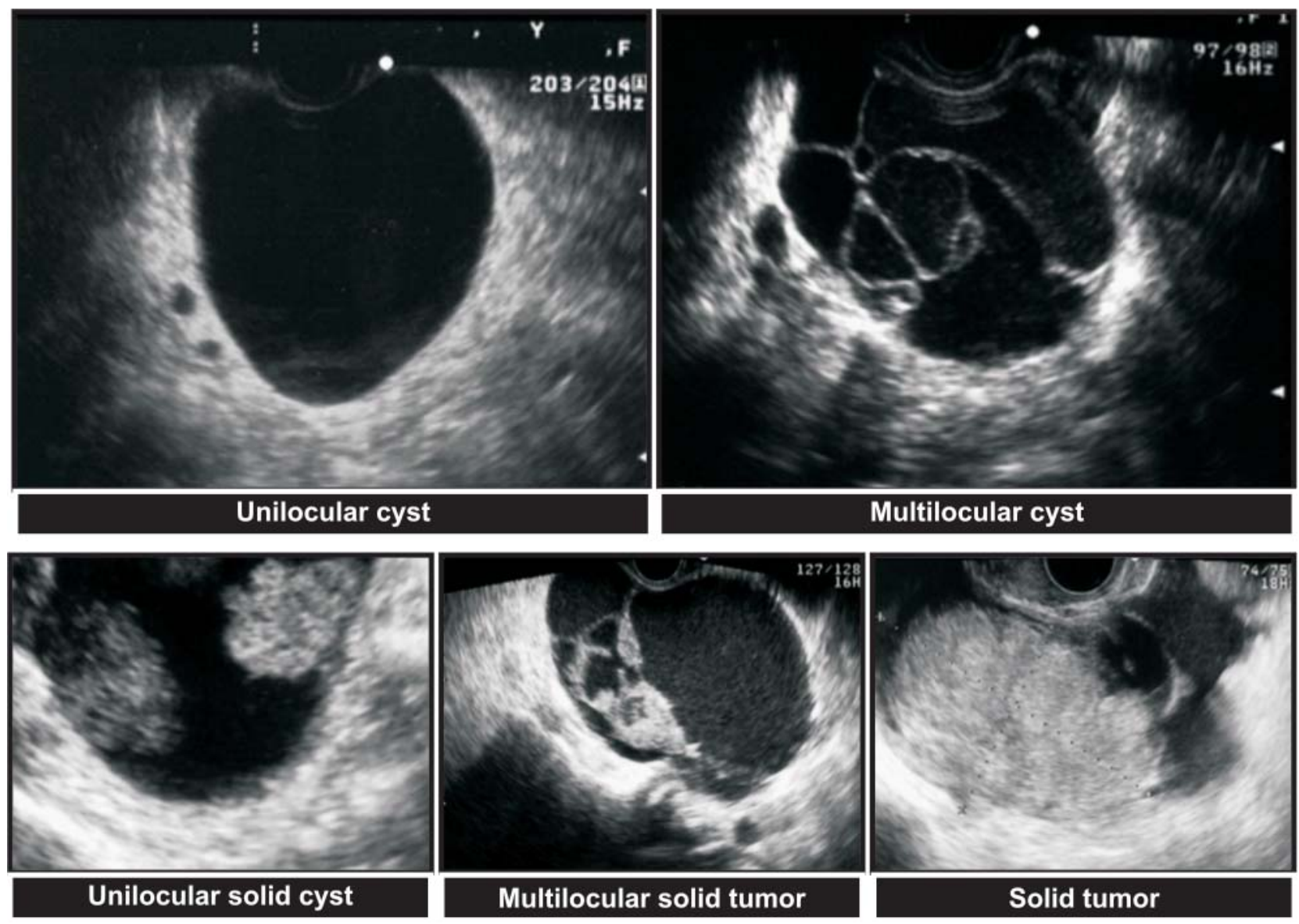

Fig. 3: Gray-scale ultrasonographic classification of adnexal tumor 


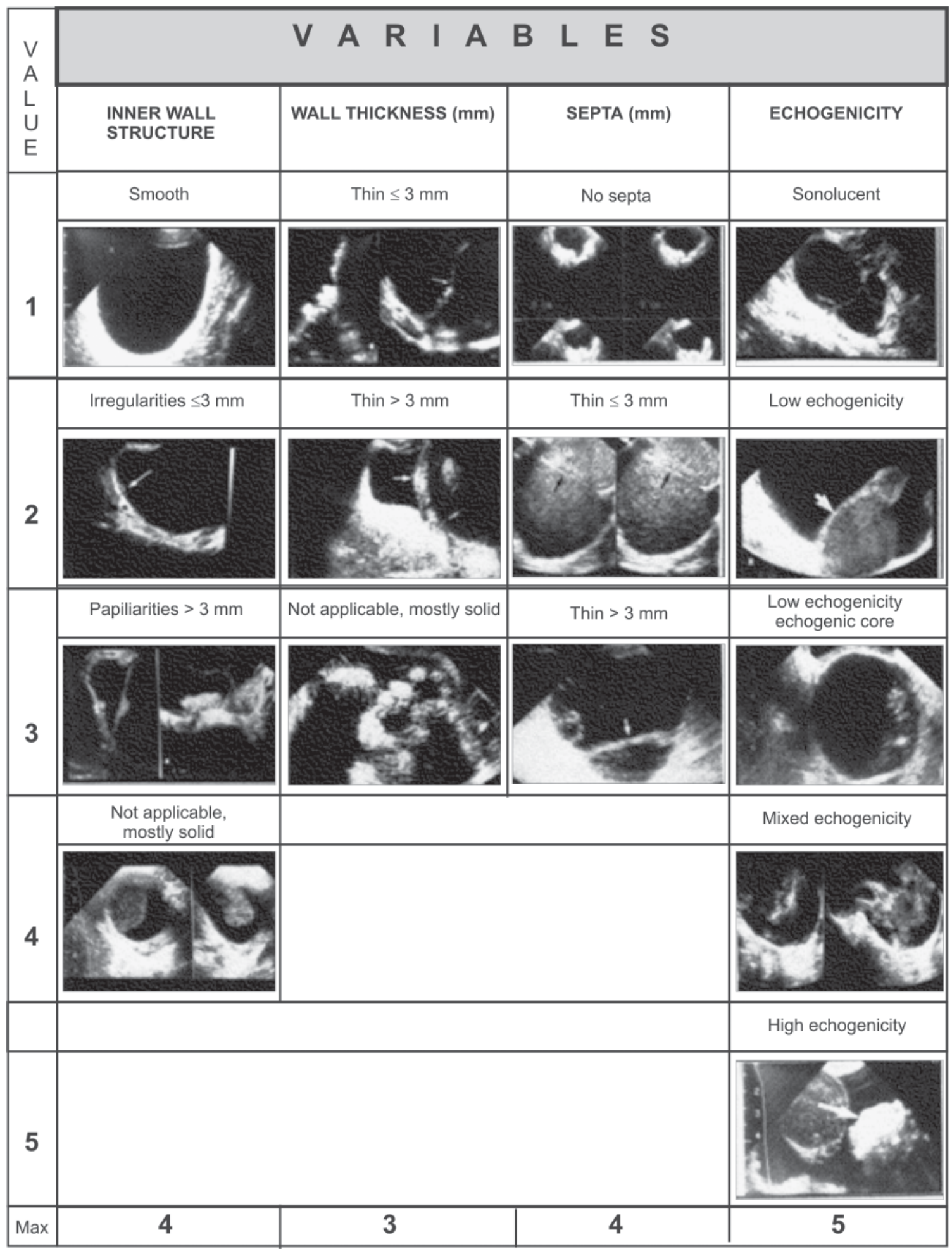

Fig. 4: Scoring system for evaluation of adnexal tumor (quoted from reference 5) 


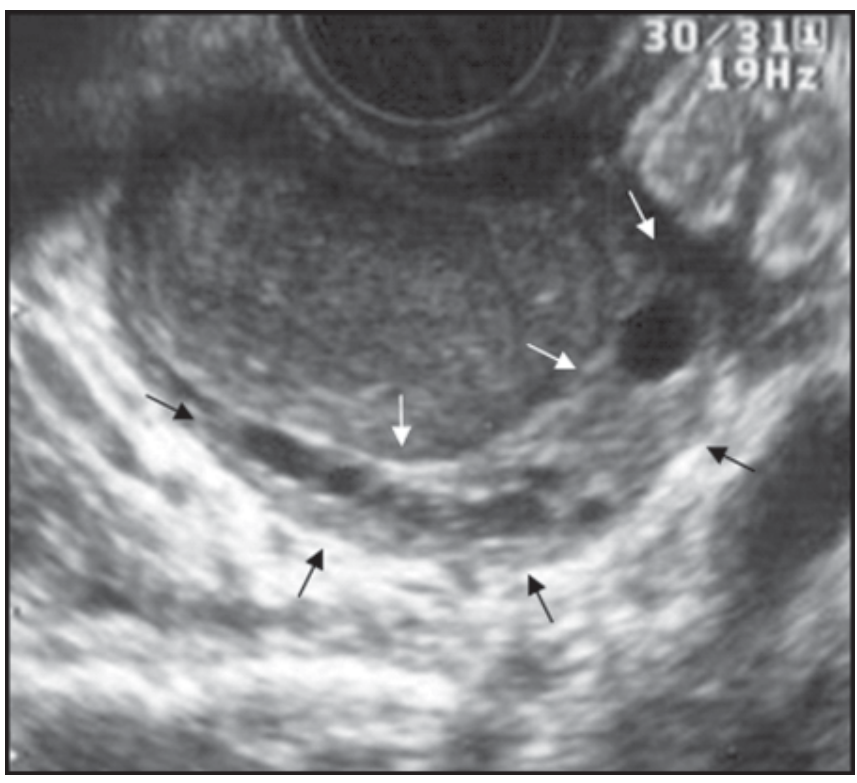

Fig. 5: 'Ovarian crescent sign' (arrows)

Recently, Hillaby et $a l^{8}$ reported that the presence of normal ovarian tissue (the 'ovarian crescent sign') adjacent to an ovarian cyst (Fig. 5) is a useful morphological feature that may be used to help exclude an invasive ovarian malignancy in women with adnexal masses detected on ultrasound scan. Using this method, i.e. the 'ovarian crescent sign', the differential diagnosis of an ovarian mass is based on the identification of normal ovarian tissue surrounding the tumor, rather than on the detailed morphological examination of the tumor itself. Malignant ovarian lesions could be diagnosed with a sensitivity of $91 \%$ and specificity of $84 \%$ in 100 women with a preoperative diagnosis of an adnexal mass, which seemed to be relatively proper. However, further prospective work might be necessary to assess the potential value of the 'ovarian crescent sign.'

\section{Transvaginal Color and Pulsed Doppler Diagnosis}

The introduction of transvaginal Doppler ultrasonography into gynecologic field has allowed detailed hemodynamic assessment of ovarian tumors. The presence or absence of color and the detection or non-detection of flow velocity waveforms could be used as categorical endpoints (which depend on the presence of high blood velocity). The resistance index (RI) or pulsatility index (PI) was used as continuous variables; these values are highly dependent upon diastolic velocity and are thought to reflect impedance distal to the point of sampling. The use of these endpoints in relation to derived cut-off values gave encouraging results for distinguishing between benign and malignant tumors. Kurjak et $a l^{9}$ stressed the importance of thorough examination of ovaries with transvaginal color Doppler regardless of their B-mode ultrasound appearance or size, and found a resistance index (RI) of less than 0.4 in only one of 624 benign tumors, whereas an RI of less than 0.4 was found in 54 of 56 malignant tumors. They set the cut-off value of RI, 0.40 . Sequentially, some authors arbitrarily set the cut-off value of RI or PI with excellent sensitivity and specificity. ${ }^{10-12}$ However, we were unable to discriminate between the blood flow of a corpus luteum cyst, an endometrioid ovarian cyst and carcinoma, and suggested that we had yet to determine the values that define normal and abnormal blood flow within the ovary. ${ }^{13}$

When ultrasound equipment became more sensitive (in terms of detecting lower blood velocity), more areas of color, flow velocity waveforms and vessels with low RI and PI were found in benign tumors, and the technique did not appear to offer significant advantages over B-mode imaging. ${ }^{14,15}$ Paradoxically, the advance in technology was a contributory factor in momentarily reducing the usefulness of the technique for detecting ovarian cancer. There was renewed interest in the potential use of color Doppler imaging for this purpose; however, when it was found that reproducible measurements could be made of the peak systolic velocity (PSV) in ovarian follicles and corpora lutea, ${ }^{16}$ and variety of overt tumors. ${ }^{17}$

The accurate measurement of blood velocity is dependent upon the angle of the ultrasound beam to the vessel. Unfortunately, it is not possible to determine the angle of insonation to small intratumoral vessels. However, the volume of tissue under the range gate is large enough to include many vessels, which may be arranged in a tortuous manner. It has been generally accepted that one or more of these small vessels will be at a low or zero angle to measurements can be made. We have shown that the intratumoral PSV is the most useful index for discriminating between overt benign and malignant adnexal masses. ${ }^{18}$ This conclusion was reinforced by the findings of Tailor et al. ${ }^{17}$ that the intratumoral PSV and time-averaged maximum velocity (TAMXV) were better indices than either the $\mathrm{RI}$ or PI and that the combined use of one index for velocity and another for impedance was more useful than using either alone.

Now, the situation becomes even more complex when using transvaginal color Doppler imaging with pulsed Doppler spectral analysis. Differences in operator variance and sensitivity contribute to a confusing analysis of variables. In some studies, Doppler ultrasound evaluation is still helpful in the diagnosis of adnexal malignancy. ${ }^{19,20}$ On the other hand, Doppler flow studies did not improve diagnostic accuracy. ${ }^{21}$

\section{Multivariate Logistic Regression Analysis}

Multivariate logistic regression analysis is a mathematical procedure for systematically developing an algorithm to assess the probability that a particular event has occurred. This approach has been used to determine the probability of nodal involvement in patients with prostatic carcinoma based on the 
patients' age, the serum acid phosphatase level, X-ray findings, tumor grade. ${ }^{22}$ Tailor et $a l^{23}$ assigned a probability of malignancy for any patient with an adnexal tumor by the application of multivariate logistic regression analysis to variables recorded at the time of pelvic sonography. Multivariate logistic regression analysis enabled the calculation of probability of malignancy for any patient with a known adnexal mass, and ensured reproducibility of diagnosis reduce dependence on operator experience. The accuracy of that prediction appeared to be better than that of morphological or Doppler criteria when the latter were used independently. Another important advantage was the ability to estimate the probability of malignancy that was helpful in both decision making and patient counseling. ${ }^{23-26}$ However, these published studies were designed using a limited sample size, and therefore must be tested on a large number of women before then can be introduced into clinical practice. Recently, based on the data from 1,066 patients recruited from nine European centers, a more universally useful regression model to distinguish between a malignant and benign adnexal tumor before surgery has been developed. ${ }^{27}$ Because the model was constructed from multicenter data, it is more likely to be generally applicable. However, I still wonder whether the terms, definition, and qualitative and quantitative endpoints to describe the ultrasonic features of adnexal tumors were being really used in the protocol for those studies with specific objectives. Are internationally agreed terms and definition necessary? I take precautions against these steering studies of mathematical game performed in western countries.

\section{SUBJECTIVE ASSESSMENT}

Timmerman et $a l^{27}$ stated that most ultrasonographers base their interpretation of ultrasound images on a subjective estimation rather than on the use of decision levels or scoring systems. They concluded that subjective evaluation of the grayscale ultrasound image, i.e. pattern recognition, for discrimination between benign and malignant tumors can almost certainly be learnt by anyone performing gynecological ultrasound examinations on a regular basis, but diagnostic accuracy increases with increasing experience. Pattern recognition has been shown to be superior to all other ultrasound methods (e.g. simple classification system, scoring system, mathematical models for calculating the risk of malignancy) for discrimination between benign and malignant extrauterine pelvic masses. Adding Doppler ultrasound examination to subjective evaluation of the gray-scale ultrasound image does not seem to yield much improvement in diagnostic precision, but it may increase the confidence with which a correct diagnosis of benignity or malignity is made. ${ }^{28-30}$ Certain tumors (e.g. endometriosis, dermoid cysts, hydro- pyo- and hematosalpinx, peritoneal pseudocysts, paraovarian cysts, hemorrhagic corpus luteum cysts, myomas, abscesses and ovarian fibromas, thecomas, and Brenner tumors) may manifest a characteristic appearance at gray-scale imaging (Figs 6 to 10). Therefore, an experienced ultrasound examiner can often make a correct specific diagnosis based on the grayscale ultrasound image by pattern recognition. ${ }^{31}$ However, in some case, the diagnosis with pattern recognition may be confusing and the following observations might be clinically helpful in such case ${ }^{32-34}$ (Figs 11 and 12).

1. Simple cysts and developing follicles usually exhibit minimal or no flow in their walls. ${ }^{32}$

2. The preovulatory follicle presents a large amount of flow in its wall. ${ }^{33}$

3. Recent (hemorrhagic) corpus luteum cysts tend to have a very strong peripheral (circular) flow. ${ }^{32}$

4. Endometrial cysts most often present a very low and localized flow. ${ }^{34}$

5. Benign cystic teratoma rarely exhibit internal blood flow. ${ }^{32}$

6. Most other benign ovarian tumors present a low or moderate perfusion in their walls and septa. ${ }^{32}$

7. Pelvic inflammatory disease (PID) is associated with very strong blood flow in the septa and cyst wall. ${ }^{32}$

8. Ovarian cancer commonly exhibits irregular and very strong blood flow in the septa and also in the cyst wall. ${ }^{32}$

\section{NEW TRENDS}

Three-dimensional power-Doppler imaging (3DPD) has become available. Several studies have shown that 3DPD might improve the diagnostic accuracy for ovarian cancer prediction. ${ }^{35-37} \mathrm{On}$

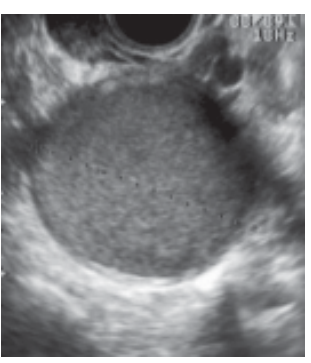

A

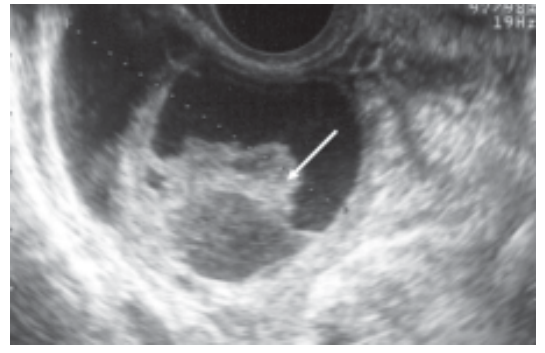

B

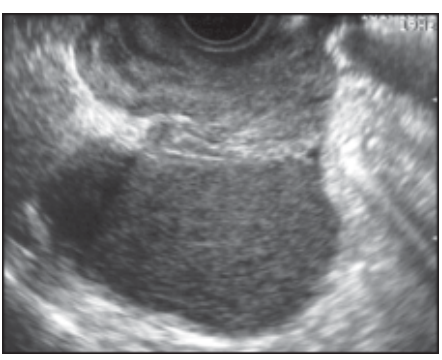

C

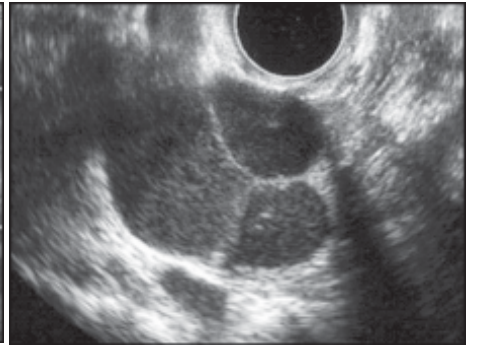

D

Fig. 6: Endometriosis with (A) fine granular echo, (B) 'wall nodularity' suggesting coagulation (arrow),

(C) adhesion to the uterus, and (D) multilocular cyst 

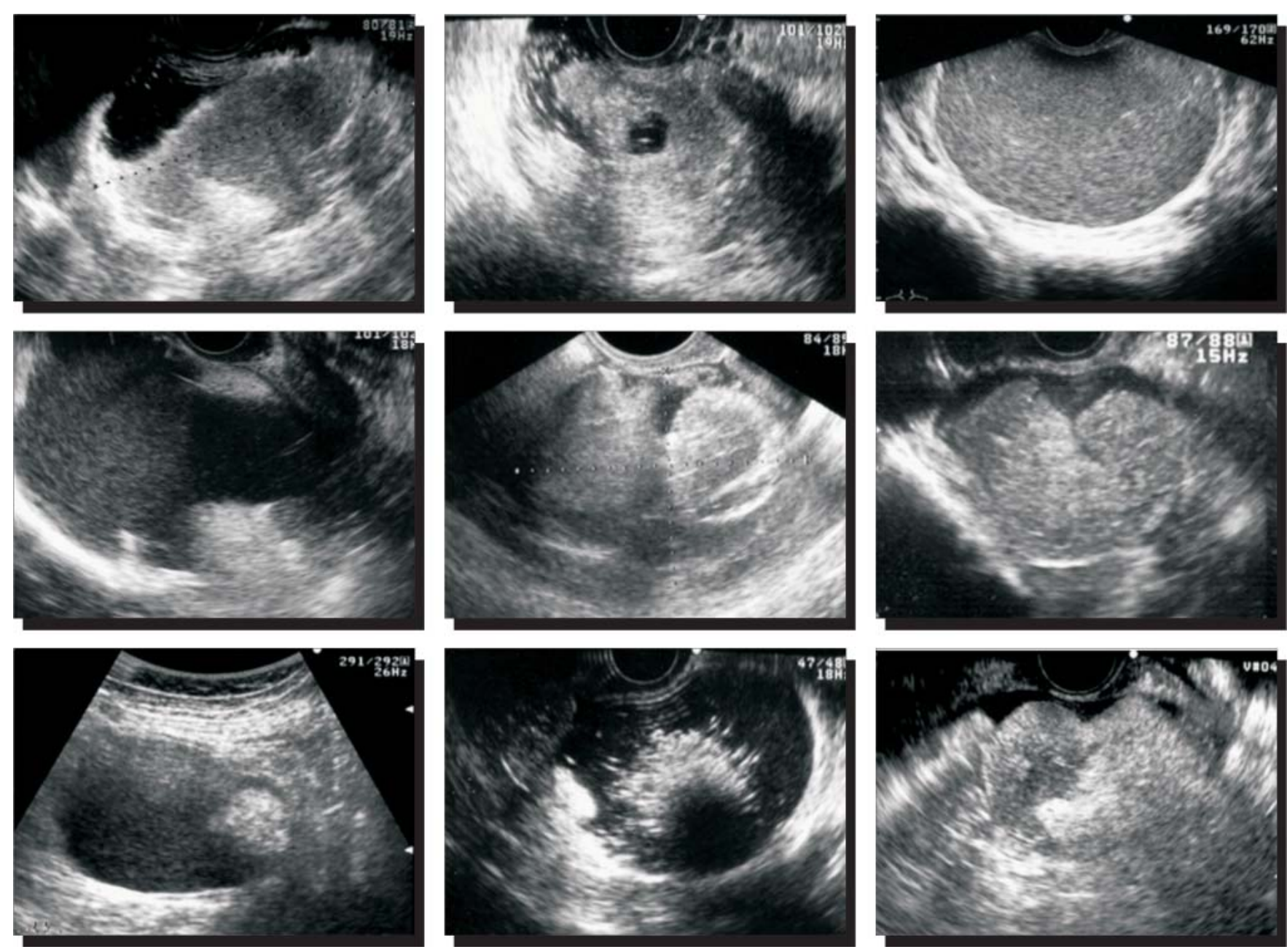

Fig. 7: Typical figures of dermoid cysts
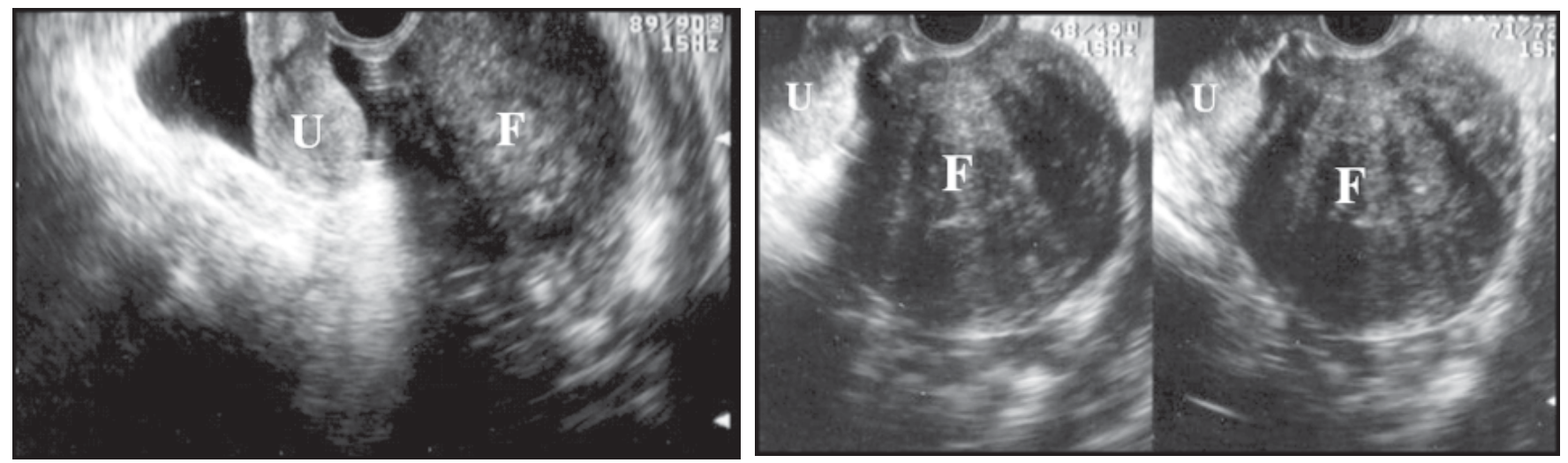

Fig. 8: A fibroma (F) may be confused with a uterine myoma if no connection is seen between the myoma and the uterus $(U)$ 


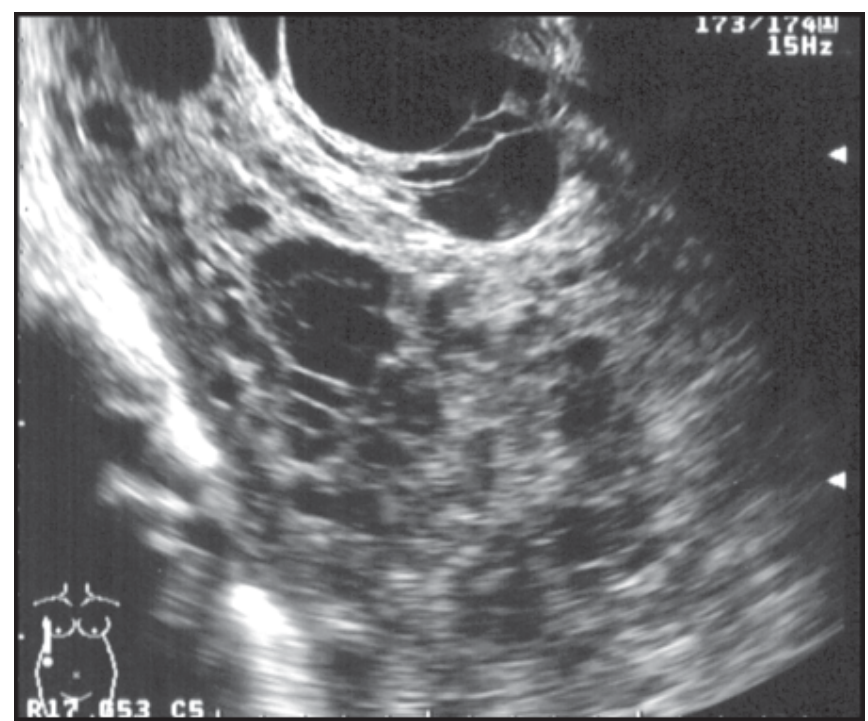

Fig. 9: A granulosa cell tumor presents honey-comb appearance

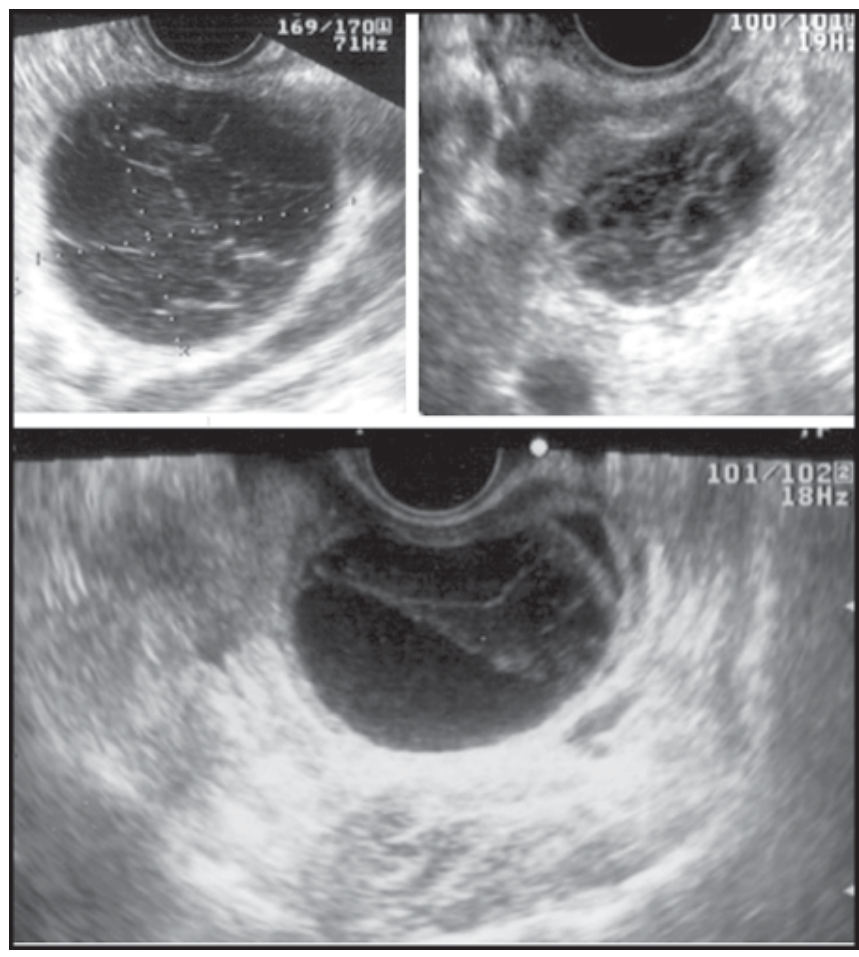

Fig. 10: A hemorrhagic corpus luteum cyst is easily diagnosed by spider-web-like contents

the other hand, Alcazar et $\mathrm{l}^{38}$ reported that 3DPD did not have a better diagnostic performance than 2-dimensional powerDoppler imaging for the discrimination of benign from malignant complex adnexal masses. We have as yet been unable to specify objective parameters in this regard. Further research is seemed to be needed to give 3DPD technique its appropriate role in the diagnostic procedure.
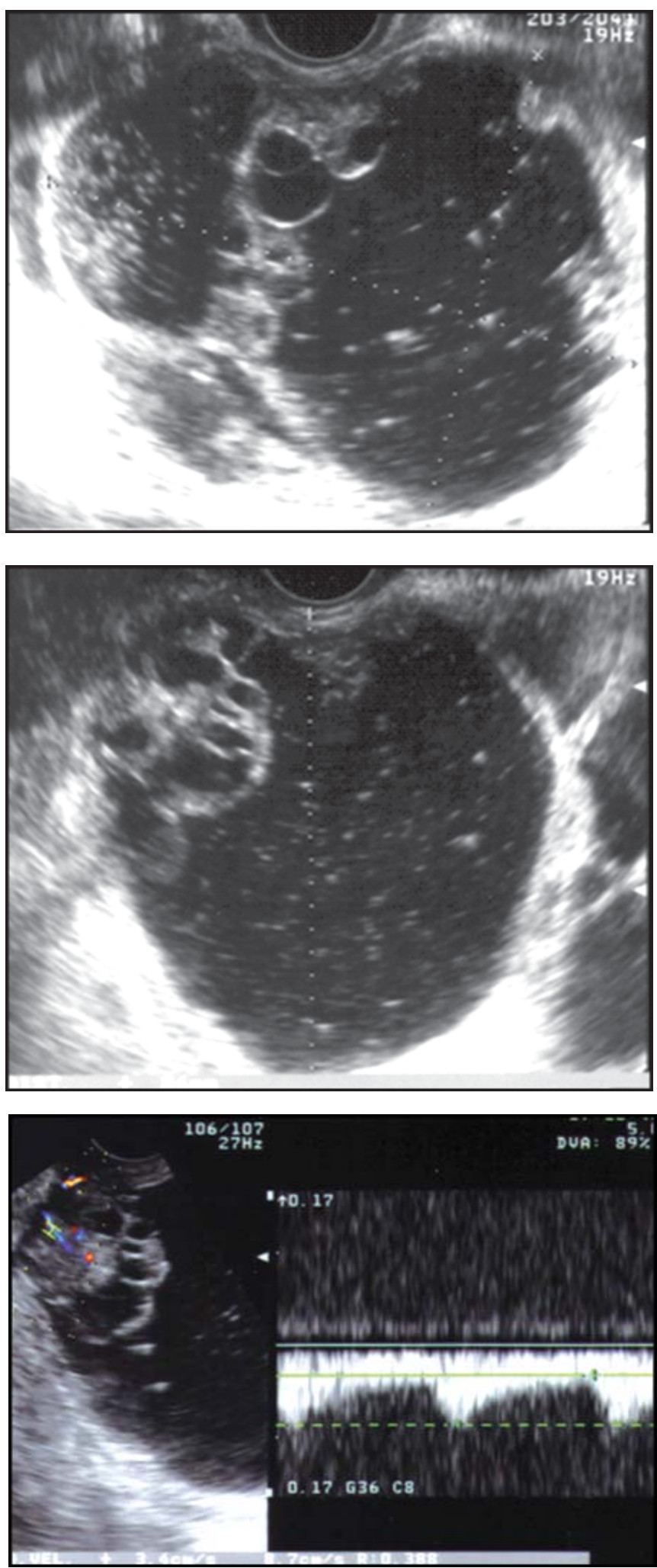

Fig. 11: By gray-scale image, this tumor might be diagnosed to be malignant. Low perfusion within solid part was recorded. This tumor was to be mucinous cystadenoma 

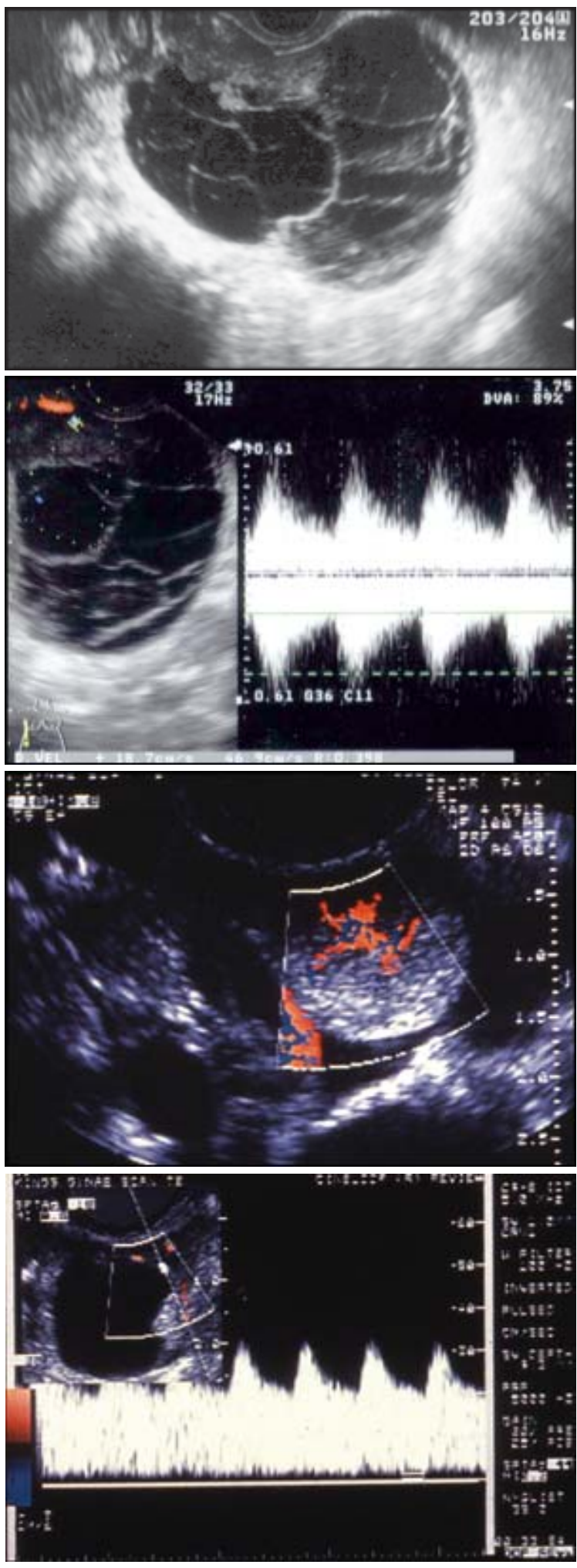

Fig. 12: Doppler ultrasonography of serous cystadenocarcinomas. Irregular and very strong blood flow in the solid component was recorded

\section{REFERENCES}

1. Bhan V, Amso N, Whitehead MI, Campbell S, Royston P, Collins WP. Characteristics of persistent ovarian masses in asymptomatic women. Br J Obstet Gynaecol 1989;96:1384-91.

2. Bourne TH, Campbell S, Steer C, Whitehead MI, Collins WP. Transvaginal color flow imaging: a possible new screening technique for ovarian cancer. Br Med J 1989;299:1367-70.

3. Granberg S, Norstrom A, Wikland M. Tumors in the lower pelvis as imaged by vaginal sonography. Gynecol Oncol 1990;37:224-9.

4. Rottem S, Levit N, Thaler I, Yoffe N, Bronshtein M, Manor D, Brandes JM. Classification of ovarian lesions by high-frequency transvaginal sonography. J Clin Ultrasound 1990;18:359-63.

5. Sassone AM, Timor-Tritsch IE, Artner A, Westhoff C, Warren WB. Transvaginal sonographic characterization of ovarian disease: evaluation of a new scoring system to predict ovarian malignancy. Obstet Gynecol 1991;78:70-6.

6. DePriest PD, Shenson D, Fried A, Hunter JE, Andrews SJ, Gallion HH, Pavlik EJ, Kryscio RJ, van Nagell JR Jr. A morphology index based on sonographic findings in ovarian cancer. Gynecol Oncol 1993;51:7-11.

7. Lerner JP, Timor-Tritsch IE, Federman A, Abramovich G. Transvaginal ultrasonographic characterization of ovarian masses with an improved, weighted scoring system. Am J Obstet Gynecol 1994;170:81-5.

8. Hillaby K, Aslam N, Salim R, Lawrence A, Raju KS, Jurkovic $D$. The value of detection of normal ovarian tissue (the 'ovarian crescent sign') in the differential diagnosis of adnexal masses. Ultrasound Obstet Gynecol 2004;23:63-7.

9. Kurjak A, Zalud I, Alrfevic A. Evaluation of adnexal masses with transvaginal color ultrasound. J Ultrasound Med 1991;10:295-7.

10. Fleischer AC, Rodgers WH, Rao BK, Kepple DM, Worell JA, Williams L, Jones III HW. Assessment of ovarian tumor vascularity with transvaginal color Doppler sonography. J Ultrasound Med 1991;10:563-8.

11. Weiner Z, Thaler I, Beck D, Rottem S, Deutsch M, Brandes JM. Differentiating malignant from benign ovarian tumors with transvaginal color flow imaging. Obstet. Gynecol 1992;79:15962.

12. Kawai M, Kano T, Kikkawa F, Kikkawa F, Maeda O, Oguchi $\mathrm{H}$, Tomoda Y. Transvaginal Doppler ultrasound with color flow imaging in the diagnosis of ovarian cancer. Obstet Gynecol 1992;79:163-7.

13. Hata K, Makihara K, Hata T, Takahashi K, Kitao M. Transvaginal color Doppler imaging for hemodynamic assessment of reproductive tract tumors. Int J Gynecol Obstet 1991;36:301-8.

14. Hata K, Hata T, Manabe A, Sugimura K, Kitao M. A critical evaluation of transvaginal Doppler studies, transvaginal sonography, magnetic resonance imaging, and CA 125 in detecting ovarian cancer. Obstet Gynecol 1992;80:922-6.

15. Tekay A, Jouppila P. Validity of pulsatility and resistance indices in classification of adnexal tumors with transvaginal color Doppler ultrasound. Ultrasound Obstet Gynecol 1992;2:338-44. 
16. Collins W, Jurkovic D, Bourne T, Kurjak A, Campbell S. Ovarian morphology, endocrine function and intra-follicular blood flow during the peri-ovulatory period. Hum Reprod 1991; 6:319-24.

17. Tailor A, Jurkovic D, Bourne TH, Natucci M, Collins WP, Campbell S. A comparison of intratumoral indices of blood flow velocity and impedance for the diagnosis of ovarian cancer. Ultrasound Med Biol 1996;22:837-43.

18. Hata K, Hata T, Kitao M. Intratumoral peak systolic velocity as a new possible predictor for detection of adnexal malignancy. Am J Obstet Gynecol 1995;172:1496-500.

19. Erdogan N, Ozcelik B, Serin IS, Akgun M, Ozturk F. Doppler ultrasound assessment and serum cancer antigen 125 in the diagnosis of ovarian tumors. Int J Gynecol Obstet 2005;91:146-50.

20. Guerriero S, Ajossa S, Garau N, Piras B, Paoletti AM, Melis GB. Ultrasonography and color Doopler-based triage for adnexal masses to provide the most appropriate surgical approach. Am J Obstet Gynecol 2005;192:401-6.

21. Ueland FR, DePriest PD, Pavlik EJ, Kryscio RJ, van Nagell Jr JR. Preoperative differentiation of malignant from benign ovarian tumors: the efficacy of morphology index and Doppler flow sonography. Gynecol Oncol 2003;91:46-50.

22. Brown BWJ. Prediction analyses for binary data. In Miller RG, Efron B, Brown BWJ, Moses LE (Eds): Biostatistics Casebook. New York: John Wiley \& Sons 1980; pp 3-18.

23. Tailor A, Jurkovic D, Bourne TH, Collins WP, Campbell S. Songraphic prediction of malignancy in adnexal masses using multivariate logistic regression analysis. Ultrasound Obstet Gynecol 1997;10:561-7.

24. Tailor A, Jurkovic D, Bourne TH, Collins WP, Campbell S. Sonographic prediction of malignancy in adnexal masses using an artificial neural network. Br J Obstet Gynecol 1999;106:2130.

25. Timmerman D, Bourne TH Tailor A, Collins WP, Verrelst H, Vandenberghe K, Vergote I. A Comparison of methods for preoperative discrimination between malignant and benign adnexal masses: the development of a new logistic regression model. Am J Obstet Gynecol 1999;181:57-65.

26. Aslam N, Banerjee S, Carr JV, Savvas M, Hooper R, Jurkovic D. Prospective evaluation of logistic regression models for the diagnosis of ovarian cancer. Obstet Gynecol 2000;96:75-80.

27. Timmerman D, Schwarzler P, Collins WP, Claerhout F, Coenen M, Amant F, Vergote I, Bourne TH. Subjective assessment of adnexal masses with the use of ultrasonography: an analysis of interobserver variability and experience. Ultrasound Obstet Gynecol 1999;13:11-6.

28. Jain KA. Prospective evaluation of adnexal masses with endovaginal gray-scale and duplex and color Doppler US: Correlation with pathologic findings. Radiology 1994;191:63-7.

29. Valentin L. Prospective cross-validation of Doppler ultrasound examination and gray-scale ultrasound imaging for discrimination of benign and malignant pelvic masses. Ultrasound Obstet Gynecol 1999;14:273-83.

30. Valentin L, Hagen B, Tingulstad S, Eik-Nes S. Comparison of 'pattern' and logistic regression models for discrimination between benign and malignant pelvic masses; a prospective cross validation. Ultrasound Obstet Gynecol 2001;18:357-65.

31. Valentin L. Use of morphology to characterize and manage common adnexal masses. Best Pract Res Clin Obstet Gynaecol 2004; 18:71-89.

32. Timmerman D, Lack of standardization in gynecological ultrasonograpy. Ultrasound Obstet Gynecol 2000;16:395-8.

33. Campbell S, Bourne TH, Waterstone J, Reynolds KM, Crayford TJ, Jurkovic D, Okokon FV, Collins WP. Transvaginal color blood fow imaging of the periovulatory follicle. Fertil Steril 1993;60:433-8.

34. Guerriero S, Ajossa S, Mais V, Risalvoto A, Lai MP, Melis GB. The diagnosis of endometriomas using colour Doppler energy imaging. Hum Reprod 1998;13:1691-5.

35. Kurjak A, Kupesic S, Anic T, Kosuta D. Three-dimensional ultrasound and power Doppler improve the diagnosis of ovarian lesions. Gynecol Oncol 2000;76:28-32.

36. Cohen LS, Escobar PF, Scharm C, Glimco B, Fishman DA. Three-dimensional ultrasound power Doppler improves the diagnostic accuracy for ovarian cancer prediction. Gynecol Oncol 2001;82:40-8.

37. Fleischer AC, Milam MR, Crispens MA, Shappell HW. Sonographic depiction of intratumoral vascularity with 2- and 3-dimensional color Doppler techniques. J Ultrasound Med 2005;24:533-7.

38. Alcazar JL, Castillo G. Comparison of 2-dimensional and 3dimensional power-Doppler imaging in complex adnexal masses for the prediction of ovarian cancer. Am J Obstet Gynecol 2005;192:809-12. 